OPEN ACCESS

Edited by:

Leonardo Bonilha,

Medical University of South Carolina,

USA

Reviewed by:

Andras Jakab,

Medical University of Vienna,

Austria

Kaiming $L i$,

Emory University, USA

*Correspondence:

Jamie D. Feusner,

Semel Institute for Neuroscience and Human Behavior, 740 Westwood

Plaza, Room 27-469, Los Angeles,

CA 90024, USA

jfeusner@mednet.ucla.edu

Specialty section:

This article was submitted to

Neuropsychiatric Imaging and

Stimulation, a section of the journal

Frontiers in Psychiatry

Received: 02 February 2015 Accepted: 30 April 2015

Published: 20 May 2015

Citation:

Feusner JD, Moody T, Lai TM,

Sheen C, Khalsa S, Brown J, Levitt J,

Alger J and O'Neill J (2015) Brain

connectivity and prediction of relapse after cognitive-behavioral therapy in

obsessive-compulsive disorder. Front. Psychiatry 6:74.

doi: 10.3389/fpsyt.2015.00074

\section{Brain connectivity and prediction of relapse after cognitive-behavioral therapy in obsessive-compulsive disorder}

\author{
Jamie D. Feusner ${ }^{1 *}$, Teena Moody ${ }^{1}$, Tsz Man Lai ${ }^{1}$, Courtney Sheen ${ }^{1}$, Sahib Khalsa ${ }^{2,3}$, \\ Jesse Brown ${ }^{4}$, Jennifer Levitt', Jeffry Alger ${ }^{5}$ and Joseph O'Neill ${ }^{1}$ \\ ${ }^{1}$ Department of Psychiatry and Biobehavioral Sciences, University of California Los Angeles, Los Angeles, CA, USA, ${ }^{2}$ Laureate \\ Institute for Brain Research, Tulsa, OK, USA ${ }^{3}$ The University of Tulsa, Tulsa, OK, USA ${ }^{4}$ Department of Neurology, University \\ of California San Francisco, San Francisco, CA, USA, ${ }^{5}$ Department of Neurology, University of California Los Angeles, Los \\ Angeles, CA, USA
}

Background: Intensive cognitive-behavioral therapy (CBT) can effectively reduce symptoms in obsessive-compulsive disorder (OCD). However, many relapse after treatment. Few studies have investigated biological markers predictive of follow-up clinical status. The objective was to determine if brain network connectivity patterns prior to intensive CBT predict worsening of clinical symptoms during follow-up.

Methods: We acquired resting-state functional magnetic resonance imaging data from 17 adults with OCD prior to and following 4 weeks of intensive CBT. Functional connectivity data were analyzed to yield graph-theory metrics. We examined the relationship between pre-treatment connectome properties and OCD clinical symptoms before and after treatment and during a 12-month follow-up period.

Results: Mean OCD symptom decrease was $40.4 \pm 16.4 \%$ pre- to post-treatment (64.7\% responded; $58.8 \%$ remitted), but $35.3 \%$ experienced clinically significant worsening during follow-up. From pre- to post-treatment, small-worldness and clustering coefficient significantly increased. Decreases in modularity correlated with decreases in OCD symptoms. Higher pre-treatment small-world connectivity was significantly associated with worsening of OCD symptoms during the follow-up period. Psychometric and neurocognitive measures pre- and post-treatment were not significant predictors.

Conclusion: This is the first graph-theory connectivity study of the effects of CBT in OCD, and the first to test associations with follow-up clinical status. Results show functional network efficiency as a biomarker of CBT response and relapse in OCD. CBT increases network efficiency as it alleviates symptoms in most patients, but those entering therapy with already high network efficiency are at greater risk of relapse. Results have potential clinical implications for treatment selection.

Keywords: brain network, connectome, resting-state fMRI, graph theory, CBT 


\section{Introduction}

Obsessive-compulsive disorder (OCD) is characterized by recurrent, intrusive, disturbing thoughts (obsessions), and/or stereotyped recurrent behaviors (compulsions) (1). Lifetime prevalence is $1-2 \%$. Untreated OCD results in marked distress, and impaired functioning in social, occupational, and educational domains (2).

Cognitive-behavioral therapy (CBT) is an effective treatment for OCD (3). CBT typically consists of weekly outpatient treatment or intensive daily treatment over several weeks. Intensive CBT is particularly effective, resulting in sustained benefits $(4,5)$.

Notwithstanding, post-treatment relapse has been observed in roughly $20 \%$ of patients receiving CBT for OCD (6-12). Across studies, rates of relapse range widely from 0 to $50 \%$ (13). This variability may relate to differences in treatment parameters, patient characteristics, and even the definition of relapse (14). Although CBT is associated with significant symptom improvement and with lower relapse than a time-limited course of pharmacotherapy (13-19), many clinical questions remain. One important question is: what factors help predict who will remain in remission and who will relapse after treatment?

While there is some evidence for predictors of short-term response to treatment (20-22), fewer studies have addressed the equally important question of sustained response, or, conversely, worsening after treatment $(23,24)$. Several naturalistic followup studies have attempted to identify predictors of symptom course. One study followed a cohort for 21 months after a 12week randomized trial of group CBT or fluoxetine (25). Comorbid affective disorders, any comorbid psychiatric condition, and illness duration were associated with higher post-treatment OCD symptoms. A study that followed an OCD cohort treated with 12 sessions of group CBT for 2 years found that full remission of symptoms at the end of treatment protected against relapse (26). Another study followed patients for 6 years after receiving CBT with clomipramine or placebo. Greater amount of CBT, better homework compliance, and improvement at the end of treatment were associated with better outcomes (9). Likewise, in a metaanalysis of pediatric OCD, severity of illness, comorbid diagnoses, and poor initial treatment response were associated with worse longitudinal course (27). However, another study that followed patients 6-8 years after CBT plus fluvoxamine or placebo did not find an association between short-term treatment response and long-term outcome (28).

Prognostic factors that could serve as biomarkers of relapse after treatment would have potential use in clinical decisionmaking. For example, factors such as psychometric scores or neurocognitive profiles; or measurements of brain activity, connectivity, morphometry, or neurochemical profiles associated with relapse could be identified prior to a particular treatment and could be used to determine whether an alternative treatment should be pursued.

Measurement of brain activity is a logical target for identification of putative biomarkers of clinical outcome, since multiple studies associate OCD with hyperactivity in frontostriatal systems $(29,30)$. Resting-state functional magnetic resonance imaging (rsfMRI) is increasingly used to understand functional brain connectivity in psychiatric disorders. Analyses of rsfMRI data allow for examination of spontaneous fluctuations in the blood-oxygen level-dependent (BOLD) signal, revealing underlying intrinsic connectivity networks. rsfMRI studies in OCD have detected aberrant connectivity in frontostriatal and parietal regions that may contribute to the emergence and severity of OCD symptoms $(31,32)$.

Graph theory is an analysis technique used to study rsfMRI that provides information about the topology of intrinsic brain connectivity networks (33). Graph theory provides quantitative analyses of complex brain networks as a whole, rather than being limited to discrete pairs of regions. To date, there have been only two graph-theory studies of OCD. One found abnormally low connectivity in posterior temporal cortex, and abnormally high connectivity in middle cingulate, precuneus, thalamus, and cerebellum (34). A second study found abnormally low clustering coefficient, small-worldness, and local efficiency in OCD at baseline (35). Further, medication treatment was associated with increased small-worldness, clustering coefficient, local efficiency, and modularity.

The goal of the current study was to investigate, for the first time, if pre-treatment functional network connectivity measures predict worsening of OCD symptoms subsequent to CBT treatment. An additional goal was to determine the effects of intensive CBT on global brain network connectivity using graph theory. We emphasized pre-treatment rather than post-treatment values in order to uncover potential biomarkers that could provide useful input to early clinical decision-making about selection of treatment modality. We determined graph-theory metrics of rsfMRI network connectivity in individuals with OCD prior to and following 4 weeks of intensive CBT. We then followed individuals for up to 12 months post treatment, and determined the relationship between pre-treatment connectivity metrics and follow-up clinical symptomatology.

We hypothesized that intensive CBT would result in increases in functional network efficiency - mean clustering coefficient, local efficiency, and small-worldness - and modularity, based on the previous study of OCD treatment (35). In addition, we predicted a significant relationship between these graph-theory metrics pre-treatment and worsening of core OCD symptoms at follow-up. Moreover, we hypothesized that these connectivity measures, being less subjective and effort-dependent, would demonstrate stronger associations with follow-up clinical status than psychometric and neurocognitive variables.

\section{Materials and Methods}

\section{Participants}

Seventeen right-handed adults ages 21-50 diagnosed with DSM-IV OCD (36) participated. Participants were recruited from UCLA clinics, local psychiatrists and psychotherapists, flyers, and Internet advertisements. All provided written informed consent, and the UCLA Institutional Review Board approved the study. Diagnoses of OCD participants were established through detailed interviews conducted by one of the authors (JDF), who has clinical experience with this population. Primary OCD and comorbid diagnoses were determined using the ADIS-IV-Mini (37). Participants were eligible if they scored $\geq 16$ on the Yale-Brown 
TABLE 1 | Demographics of OCD participants

\section{Demographics}

Total participants $(N)$

Gender (F/M)

Age (years)

Years of education

IQ (WASI)

No comorbidities/comorbidities

Medicated/unmedicated during intensive CBT phase

Medication treatment only during follow-up phase

CBT only during follow-up phase

Medications and CBT during follow-up phase

No treatment during follow-up phase

Mean follow-up duration (mo.)

WASI, Wechsler Abbreviated Scales of Intelligence; CBT, cognitive-behavioral therapy.

Obsessive-Compulsive Scale (YBOCS) (38). Exclusion criteria included a psychotic disorder, bipolar disorder, lifetime substance dependence, or attention-deficit hyperactivity disorder. Comorbid anxiety disorders were allowed, as long as OCD was the primary diagnosis. Comorbid major depressive disorder (MDD) or dysthymic disorder was allowed, but individuals were excluded if the ADIS-IV clinical significance rating was 6 or higher (severe). Unmedicated or medicated participants were included, but serotonin reuptake inhibitors were the only class of medication allowed. In addition, they could not have had any changes (dose or agent) to their medication within 12 weeks prior to enrollment. Other exclusion criteria included IQ $<80$ on the Wechsler Abbreviated Scales of Intelligence (WASI) (39) and medical conditions that affect cerebral metabolism (e.g., thyroid disorders, diabetes). We also excluded those with prior courses of CBT for OCD consisting of $\geq 30$ sessions, in order to minimize the possibility that such patients may already have experienced putative brain changes induced by CBT. Demographics and psychometrics are reported in Tables $\mathbf{1}$ and 2, and Table S1 in Supplementary Material. Three individuals were taking stable doses of serotonin reuptake inhibitors (two fluoxetine, one escitalopram) and 14 were unmedicated.

\section{Psychometric Evaluations}

An independent evaluator, not involved in treatment or initial assessments, administered the psychometric instruments posttreatment and during the follow-up period. After 4 weeks of intensive CBT, participants returned to care as usual, which may have included medication treatment and/or psychotherapy (CBT or other), or no treatment. Thus, the follow-up period was naturalistic rather than controlled.

The primary outcome was the YBOCS (38). The YBOCS is a reliable and valid semi-structured clinical interview assessing OCD severity and change over time. It has excellent psychometric properties and sensitivity to treatment. Treatment response was defined as a $\geq 35 \%$ reduction of YBOCS total score and a Clinical Global Improvement (CGI) score of $\leq 2$; remission was defined as a YBOCS score of $\leq 14$ (40). Secondary outcome measures pre- to post-treatment included the Hamilton Anxiety Scale (HAMA) (41) and the Montgomery-Åsberg Depression Rating Scale (MADRS) (42). A general rating of functionality and of social and occupational performance was provided by the Global Assessment Scale (GAS) (43).

We also administered the Stroop Color-Word Interference Task (44) to measure response inhibition. Within this neurocognitive task, the interference trial requires the participant to inhibit a pre-potent response in order to provide a correct answer. We used interference scores as a secondary independent variable of interest.

\section{Clinical Procedures}

All participants underwent intensive CBT with an individual therapist, consisting of 90-min sessions, 5 days per week, for 4 weeks. (For additional details see Supplementary Material.)

Following treatment, participants underwent the Stroop task and psychometric evaluations. They were then followed over a period of $1-12$ months (mean $7 \pm 4.53$ ). Follow-up assessments included the YBOCS, MADRS, HAMA, the Sheehan Disability Scale (45), and the Quality of Life Enjoyment and Satisfaction Questionnaire Short Form (Q-LES-Q) (46).

\section{fMRI Acquisition}

Participants were scanned prior to and following 4 weeks of CBT. Magnetic resonance data were acquired at $3 \mathrm{~T}$ using a Siemens Trio with 12-channel headcoil. Whole-brain fMRI was collected using a 7-min echo-planar imaging (EPI) sequence (TR/TE $=2000 / 25 \mathrm{~ms}$, flip angle $=78^{\circ}$, voxels $3 \mathrm{~mm}^{3}, 35$ interleaved slices with $1-\mathrm{mm}$ gap). Participants were instructed to rest with eyes closed, to remain as still as possible, and not to sleep. High-resolution T1weighted whole-brain structural MRI was acquired using an axial MPRAGE sequence $\left(\mathrm{TR} / \mathrm{TE}=1900 / 3.26 \mathrm{~ms}\right.$, voxels $\left.1 \mathrm{~mm}^{3}\right)$, used for registration of BOLD data.

\section{rsfMRI Processing}

Functional data were preprocessed using FMRI Software Library (FSL) version 5.0.4 ${ }^{1}$. To allow for magnetization equilibrium, we discarded the first two images. Data were slice-time corrected, were motion-corrected (MCFLIRT), and band-pass filtered $(0.009-0.08 \mathrm{~Hz})$. A seven degrees-of-freedom (DOF) transform was used to register each participant's functional image to the MPRAGE, and a 12-DOF transform was used to register the MPRAGE to Montreal Neurological Institute (MNI) standard atlas space. All images were inspected for proper registration to the MNI template. Data were then resampled to $2-\mathrm{mm}$ space. White matter, CSF, and temporal derivatives were removed by linear regression, as were six head motion parameters. Motion was assessed using DVARS (root mean squared change in BOLD signal from volume to volume), and one participant was excluded whose DVARS exceeded 25\% (47). There was no difference in motion pre-and post-treatment, as indicated by DVARS measured for each subject across the time-series $(9.69 \pm 1.51$ pre-CBT and $9.29 \pm 1.16$ post-CBT; $P=0.33$ ) (Figure $S 2$ in Supplementary Material).

\section{Brain Network Extraction}

One hundred sixty functionally defined nodes were used, covering the whole brain, as previously described (48) (Figure 1). Nodes

${ }^{1}$ http://www.fmrib.ox.ac.uk/fsl 
TABLE 2 | Psychometrics of OCD participants

\begin{tabular}{|c|c|c|c|c|c|c|}
\hline Psychometric & Pre-treatment & Post-treatment & Follow-up & Statistic & df & $P$ value \\
\hline YBOCS & $23.12 \pm 3.04^{a}$ & $13.76 \pm 4.25^{\mathrm{b}}$ & $15.47 \pm 6.65^{c}$ & $F=26.62$ & $1.44,22.98$ & $<0.001$ \\
\hline HAMA & $11.94 \pm 4.78^{\mathrm{e}}$ & $8.06 \pm 4.66^{f}$ & $9.12 \pm 4.92^{\mathrm{g}}$ & $F=5.85$ & 2,32 & 0.007 \\
\hline MADRS & $12.12 \pm 5.51$ & $9.53 \pm 9.71$ & $10.00 \pm 4.86$ & $F=0.85$ & $1.43,22.89$ & 0.40 \\
\hline GAS & $59.06 \pm 6.65^{h}$ & $74.06 \pm 13.66^{i}$ & $73.65 \pm 10.95^{j}$ & $F=14.58$ & 2,32 & $<0.001$ \\
\hline Stroop interference & $54.59 \pm 7.37$ & $54.47 \pm 7.88$ & $\mathrm{~N} / \mathrm{A}$ & $t=0.10$ & 16 & 0.92 \\
\hline Sheehan (\%) & N/A & N/A & $27.25 \pm 20.56$ & $\mathrm{~N} / \mathrm{A}$ & N/A & N/A \\
\hline QLESQ (\%) & N/A & $\mathrm{N} / \mathrm{A}$ & $59.56 \pm 10.13$ & $\mathrm{~N} / \mathrm{A}$ & $\mathrm{N} / \mathrm{A}$ & $\mathrm{N} / \mathrm{A}$ \\
\hline
\end{tabular}

YBOCS, Yale Brown Obsessive-Compulsive Scale; HAMA, Hamilton Anxiety Scale; MADRS, Montgomery Åsberg Depression Rating Scale; GAS, Global Assessment Scale; Sheehan, Sheehan Disability Scale; QLESQ, Quality of Life Enjoyment and Satisfaction Questionnaire Short Form.

${ }^{a}$ vs. ${ }^{b}: P<0.001 ;{ }^{a}$ vs. ${ }^{c}: P=0.001 ;{ }^{b}$ vs. ${ }^{c}: P=0.673 ;{ }^{e}$ vs. ${ }^{f}: P=0.011 ;{ }^{e}$ vs. ${ }^{g}: P=0.079 ;{ }^{f}$ vs. ${ }^{g}: P=1 ;{ }^{h}$ vs. ${ }^{i}: P<0.001 ;{ }^{h}$ vs. ${ }^{j}: P<0.001 ;{ }^{i}$ vs. ${ }^{j}: P=1$
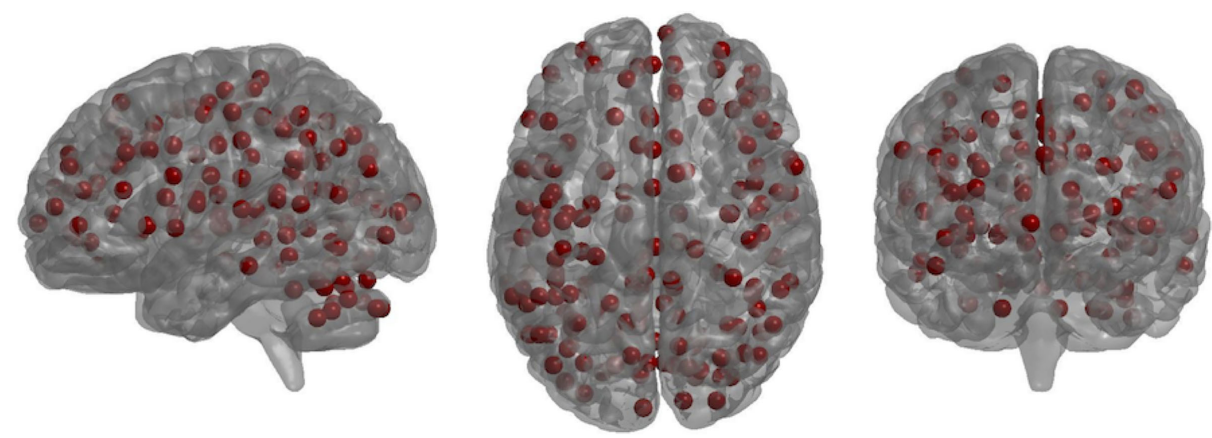

FIGURE 1 | Sagittal, axial, and coronal views of the 160 nodes used for the graph-theory analysis

were $10 \mathrm{~mm}$ in diameter and non-overlapping. Applying these nodes to each individual's rsfMRI time-series data, all pairwise partial correlation coefficients between nodes were calculated. We chose partial over full correlations as the former make topological parameters less sensitive to motion, especially at higher density thresholds (49). (We additionally performed Pearson full correlation analyses - see Supplementary Material.) Using the Brain Connectivity toolbox http://www.brain-connectivity-toolbox.net/, we derived weighted, undirected graphs, or functional connectivity matrices, for each participant.

In graph theory, a network comprises "nodes" (here, functionally defined ROIs from five meta-analyses of fMRI activation studies) and the connections or "edges" between them (in this case, partial correlation values). The clustering coefficient of a node is the ratio of the number of actual connections among its first-degree neighbors to the number of all possible such connections. Thus, a high clustering coefficient for a node indicates that its neighbors are strongly inter-connected. Mean clustering coefficient is the average of the clustering coefficient for all nodes in the network; high values may confer greater local efficiency of information transfer in a network (33). Global efficiency is mathematically defined by averaging the inverse shortest path lengths across all node pairs. (Path length is the minimum number of intermediate nodes needed to pass through in order to link any node pair.) High global efficiency represents high overall capacity for parallel information transfer and integrated processing (50). Small-worldness is mean clustering coefficient divided by characteristic path length. Modularity measures how strongly nodes in a community interconnect in comparison to nodes in a random graph. Thus, the higher the modularity of a given community structure, the less likely it is the result of chance alone. Modularity quantifies the degree to which the network may be subdivided into such clearly delineated modules. In the brain, balance between segregation of specialized systems (modules) and integration across systems is essential for efficient information processing and rapid information transfer within and between networks $(51,52)$.

From the connectivity matrices, we calculated small-world parameters (including mean clustering coefficient, smallworldness, local and global efficiencies), and modularity, to examine global network topologies. For small-world analysis, we used the sparsity threshold $S$ to define the small-world regime $(0.10 \leq S \leq 0.50)$. Each small-world attribute was compared with those of 100 random networks to produce a normalized value, and areas under the curve (AUC) were calculated for statistical comparisons.

\section{Statistical Analyses}

We tested relationships between pre-treatment graph-theory network measures and change in OCD symptoms during follow-up using multiple linear regression in SPSS ${ }^{\circledR}$. All data were checked for normality of distributions and for outliers. We tested smallworldness, mean clustering coefficient, global efficiency, local efficiency, and modularity. The primary outcome was change in YBOCS from post-treatment to the follow-up time point. For the prediction of follow-up score analyses, we controlled for the number of months post-treatment at which follow-up data were acquired, as well as for number of months of medication treatment 
and number of CBT sessions (if any) in the follow-up period, and for motion (DVARS for the pre-CBT scan). Because smallworldness is a function of clustering coefficient and (the inverse of) global efficiency, and local efficiency and clustering coefficient are related and therefore non-independent, we use a Bonferronicorrected $\alpha$ threshold of $0.05 / 3=0.017$ for the independent statistical metrics (clustering coefficient, global efficiency, and modularity).

Secondary analyses examined whether clinical (HAMA and MADRS scores) or neurocognitive (Stroop interference scores) variables pre- or post-treatment significantly predicted follow-up YBOCS score changes. A Bonferroni-corrected $\alpha$ threshold of $0.05 / 6=0.0083$ was used for statistical significance.

To assess differences pre- to post-treatment within-group, we compared AUC values obtained across the range of sparsities. We applied multiple linear regression before computation to remove the confounding effects of motion (DVARS) for each graph-theory metric. We performed paired $t$-tests, or one-sample permutation tests in $\mathrm{R}^{2}$, for data that were normally or non-normally distributed, respectively. A significance threshold of $\alpha=0.017$, two-tailed, was applied, as above.

As exploratory analyses, we tested for pre- to post-CBT changes at the level of individual regions for all 160 nodes, for the graphtheory metrics node degree, clustering coefficient, local efficiency, betweenness centrality, and edge betweenness centrality. We used false discovery rate (FDR) to correct for multiple comparisons for each metric, with a $q$ value set at. $05 / 4=0.0125$ (to account for four independent tests of metrics, as clustering coefficient and local efficiency are related) as the significance threshold.

\section{Results}

Treatment Response and Follow-Up Clinical Data Follow-up data were available for all 17 participants. Mean duration of follow-up was $7 \pm 4.53$ months. Four participants received medication only in the follow-up period, eight were treated with CBT only, two with medications and CBT, and three received no treatment (Table 1).

All 17 participants completed treatment. Mean YBOCS scores decreased $40.4 \pm 16.4 \%$ from pre- to post-treatment $[t(16)=10.00, P<0.0001]$ (Table 2). Eleven (64.7\%) were responders and $10(58.8 \%)$ achieved remission.

There was a mean increase in YBOCS from post-treatment to follow-up of $13.98 \pm 50.91 \%$. Six (35.3\%) experienced clinically significant worsening of symptoms during follow-up consisting of $\geq 5$ points worsening on YBOCS (53).

\section{Pre- to Post-Treatment Changes in Graph-Theory Global Network Metrics}

Small-worldness AUC significantly increased from pre- to post-treatment $(P=0.012)$, as did mean clustering coefficient $(P=0.0093)$ (Figures 2 and 3$)$. There were non-significant increases in local efficiency $(P=0.070)$, non-significant decreases in modularity $(P=0.12)$, and non-significant decreases in global efficiency $(P=0.18)$. Post hoc analyses excluding medicated

${ }^{2}$ http://www.r-project.org/ individuals resulted in similar significant findings, with the exception that modularity significantly decreased from pre- to posttreatment $(P=0.012)$ (see Supplementary Material).

Changes in small-worldness were relatively consistent across the sample, with 16 of 17 participants demonstrating increases pre- to post-treatment (although 2 were minimally changed) (Figure 4). Mean clustering coefficient increased in 15 of 17.

As post hoc analyses, we tested partial correlations between changes in network metrics and changes in OCD symptom scores, controlling for motion (mean pre- and post-CBT DVARS). We used a Bonferroni-corrected $\alpha$ threshold of $0.05 / 3=0.0167$ for statistical significance. Change in modularity was significantly correlated with change in YBOCS from pre- to post-treatment $(r=0.64 ; P=0.007)$. That is, decreases in modularity were associated with decreases in OCD symptoms. Correlations were nonsignificant for changes in small-worldness $(r=-0.42 ; P=0.10)$, mean clustering coefficient $(r=-0.42 ; P=0.10)$, global efficiency $(r=-0.13 ; P=0.63)$, and local efficiency $(r=-0.13$; $P=0.64)$.

\section{Exploratory Node-Level Analyses of Pre- to Post-Treatment Changes in Graph-Theory Metrics}

There were no significant differences for any nodes pre- to post-CBT for node degree, clustering coefficient, local efficiency, betweenness centrality, and edge betweenness centrality, after FDR correction (see Table S2 in Supplementary Material).

\section{Graph-Theory Metric Predictions of Follow-Up OCD Symptom Scores}

Pre-treatment small-worldness was significantly associated with follow-up change in YBOCS scores (adjusted $R^{2}=0.642$; $F_{5,11}=6.75, \quad P=0.004$ ) (Figure 5), as was pre-treatment mean clustering coefficient (adjusted $R^{2}=0.647 ; F_{5,11}=6.87$, $P=0.004)$. For both metrics, higher pre-treatment values were associated with worsening on YBOCS. Global efficiency (adjusted $R^{2}=0.21 ; \quad F_{5,11}=1.83, \quad P=0.19$ ), local efficiency (adjusted $R^{2}=0.074 ; \quad F_{5,11}=1.25, \quad P=0.35$ ), and modularity (adjusted $R^{2}=-0.032 ; \quad F_{5,11}=0.90$, $P=0.51)$ were not significant predictors of follow-up score changes.

Reanalysis of the data after excluding the three participants taking medications during the pre- to post-treatment period revealed that pre-treatment small-worldness continued to be significantly associated with follow-up change in YBOCS (adjusted $R^{2}=0.661$; $\left.F_{5,8}=6.08, P=0.013\right)$, as was pre-treatment mean clustering coefficient (adjusted $R^{2}=0.664 ; F_{5,8}=6.15, P=0.013$ ). In addition, global efficiency was associated with follow-up change in YBOCS (adjusted $R^{2}=0.593 ; F_{5,8}=4.79, P=0.025$ ), although not surviving correction for multiple comparisons; lower pretreatment global efficiency was associated with worsening on YBOCS.

As post hoc exploratory analyses, we investigated the relationships between the graph-theory metrics and additional clinical measures of anxiety (HAMA), depression (MADRS), quality of life (Q-LES-Q), and general functioning (Sheehan) at follow-up. We used a Bonferroni-corrected $\alpha$ threshold 

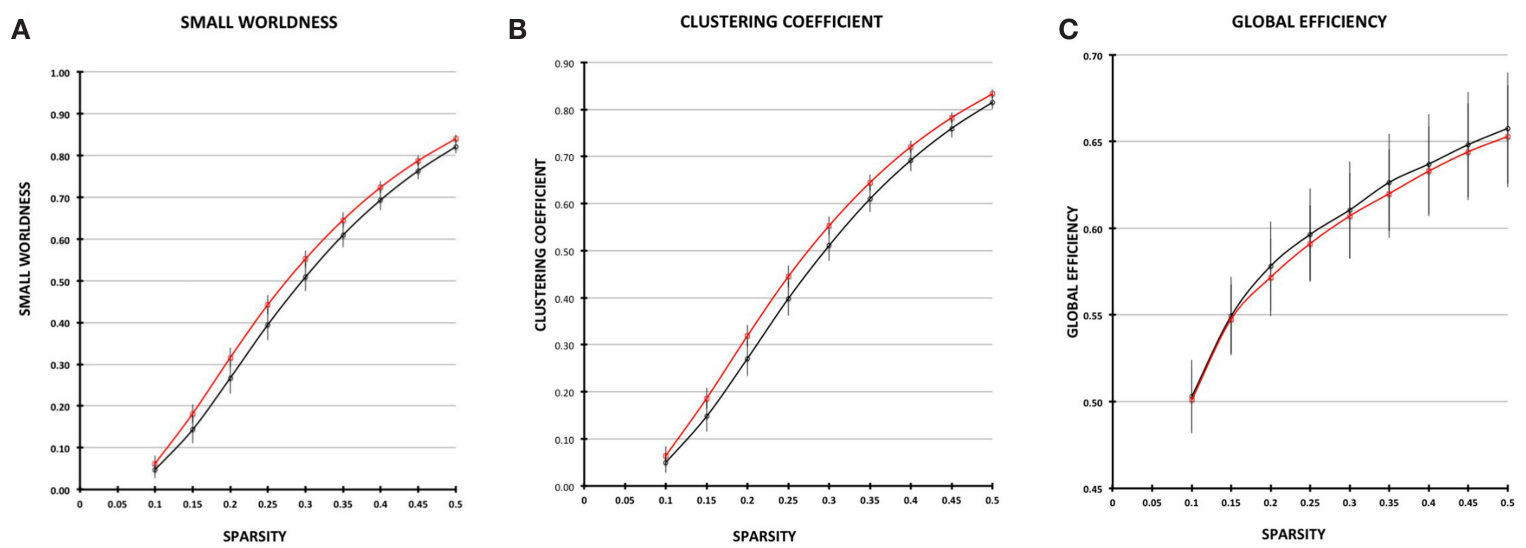

D

LCAL EFFICIENCY

E
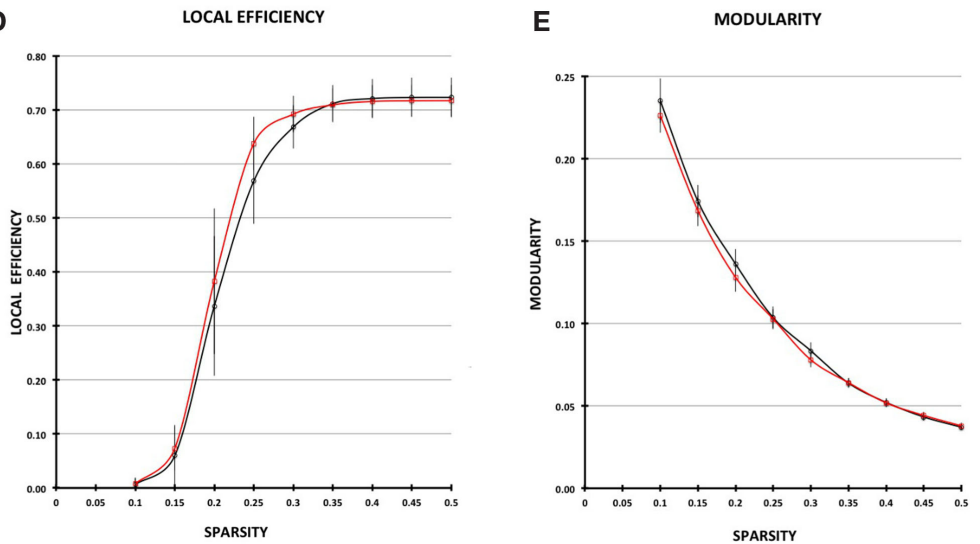

FIGURE 2 | Plot of graph-theory metrics pre- and post-treatment, derived from partial correlation matrices across sparsity levels from 0.1 to 0.5 : (A) small-worldness, (B) clustering coefficient, (C) global efficiency, (D) local efficiency, (E) modularity. Error bars are \pm 1 SD.

of $0.05 / 12=0.004$ (four clinical variables $\times$ three independent graph-theory metrics). None of the global graph-theory metrics were significantly associated with HAMA, MADRS, Q-LES-Q, or Sheehan scores.

\section{Clinical and Neurocognitive Predictors of Follow-Up OCD Symptom Scores}

Yale-Brown Obsessive-Compulsive Scale scores pre-treatment (adjusted $R^{2}=-0.002, F_{4,12}=0.99, P=0.49$ ) or post-treatment (adjusted $R^{2}=0.009, \quad F_{4,12}=1.04, P=0.43$ ) did not predict follow-up YBOCS. HAMA scores pre-treatment (adjusted $R^{2}=0.15, F_{4,12}=1.73, P=0.21$ ) and post-treatment (adjusted $\left.R^{2}=0.009, F_{4,12}=1.04, P=0.43\right)$ did not predict follow-up YBOCS scores, nor did MADRS scores pre-treatment (adjusted $R^{2}=0.002, F_{4,12}=1.01, P=0.44$ ) or post-treatment (adjusted $\left.R^{2}=0.29, \quad F_{4,12}=2.53, P=0.10\right)$. Stroop interference scores pre-treatment (adjusted $R^{2}=-0.001, F_{4,12}=0.99, P=0.45$ ) and post-treatment (adjusted $R^{2}=0.002, F_{4,12}=1.01, P=0.44$ ) did not predict follow-up YBOCS. Number of months on medications and number of follow-up CBT sessions either modeled separately (adjusted $R^{2}=-0.10, F_{2,14}=0.26, P=0.77$ for medications; adjusted $R^{2}=0.070, F_{2,14}=1.60, P=0.24$ for CBT) or together (adjusted $R^{2}=0.075, F_{3,13}=1.43, P=0.28$ ) did not significantly predict YBOCS scores.

\section{Discussion}

This is the first study to identify neuroimaging predictors of longitudinal clinical course subsequent to treatment for OCD. It is also the first to test effects of CBT treatment on brain network connectivity using graph theory. Results suggest that small-world network efficiency increases in almost all patients during CBT, but that patients with higher pre-treatment efficiency are more prone to relapse. Thus, increasing network efficiency may be CBTassociated pathways to recovery from OCD, but patients with already high network efficiency may be at greater risk of relapse of OCD symptoms.

\section{Prediction of Longitudinal Clinical Status}

As hypothesized, mean clustering coefficient and smallworldness significantly predicted follow-up OCD symptom severity, although, contrary to prediction, global efficiency and modularity did not. Mean clustering coefficient and smallworldness were better predictors than clinical measures including OCD symptom severity and anxiety and depression before or after treatment. Likewise, they performed better than a neurocognitive measure of response inhibition (Stroop), which was not significantly associated with follow-up scores. Surprisingly, months of medication and number of CBT therapy sessions in the follow-up period also did not predict OCD symptom severity. 
Thus, global measures of brain connectivity show the strongest relationship with symptom course subsequent to intensive CBT over any clinical or neurocognitive measure tested.

\section{Changes in Network Connectivity with Treatment}

As hypothesized, results demonstrated significant pre- to posttreatment increases in individuals with OCD in small-worldness and mean clustering coefficient, although increases in local efficiency were not significant. Modularity, contrary to prediction, did not significantly increase from pre- to post-treatment. These observations of increases in small-worldness and mean clustering coefficient, but no increases in global efficiency, replicate

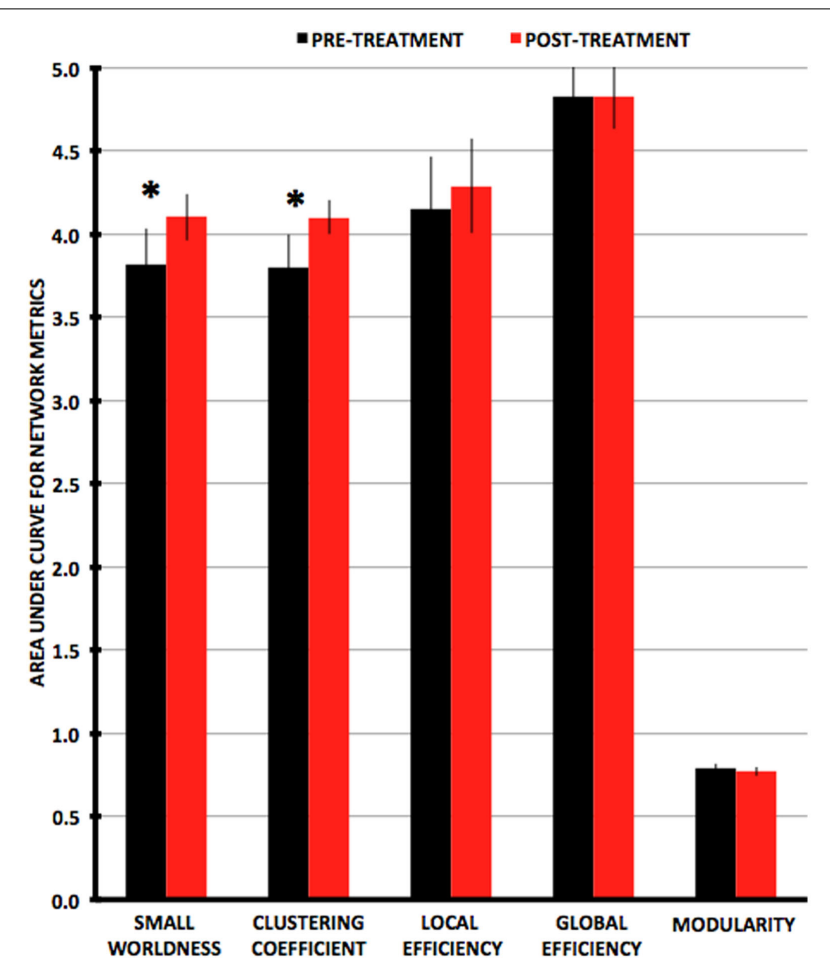

FIGURE 3 | Plots of average values for area-under-the-curve network metrics with pre-treatment in black and post-treatment in red. Metrics that are significantly different $(P<0.0167)$ pre- vs. post-treatment are indicated by an asterisk. findings in a study of OCD treatment with medication (35), although we did not observe similar changes in modularity. Therefore, there may be non-specific effects of treatment on small-world efficiency measures, but divergent effects of CBT and medications on community network structure.

Interestingly, those with lower small-worldness and lower mean clustering coefficient pre-treatment were less likely to experience worsening of their OCD symptoms in the follow-up period. These values increased significantly with treatment, although the greatest increases were for those with low pre-treatment values. This suggests that while CBT may improve network efficiency, this effect is strongest in those who start with low brain network efficiency. Moreover, these individuals do better longitudinally, in terms of sustaining or even further decreasing OCD symptom severity.

Small-world organization of brain connectivity provides for efficiency in local information processing; high local neighborhood clustering and long-distance connections offer a high degree of efficient global communication across the network and integration from different sub-specialized neighborhoods $(33,54-56)$. Since small-worldness is a function of high clustering coefficient and low characteristic path length (inverse of global efficiency), in the current study, the increases in small-world efficiency subsequent to treatment are driven by increases in mean clustering coefficient, while global efficiency remains relatively unaffected. Clustering coefficient is a measure of functional segregation; higher mean clustering coefficient means that, on average, each region's neighbors are more highly connected with each other. This can also be thought of as more densely inter-connected local networks of brain regions that confer segregated neural processing (57), for example, perhaps those involved in specialized brain functions such as attunement to specific auditory frequencies, or consolidation of fear learning in local limbic circuits. Thus, in our study, the finding of increased small-worldness as a result of increased clustering coefficient means that the brain networks have increased their overall efficiency through enhanced regional processing efficiency conferred by more dense connections, while retaining the same long distance connections (no significant changes in global efficiency) that are important for integration of information (57). Since increases in small-worldness and mean clustering coefficient were not correlated with improvements in OCD symptoms pre- to post-treatment, the mechanism of CBT

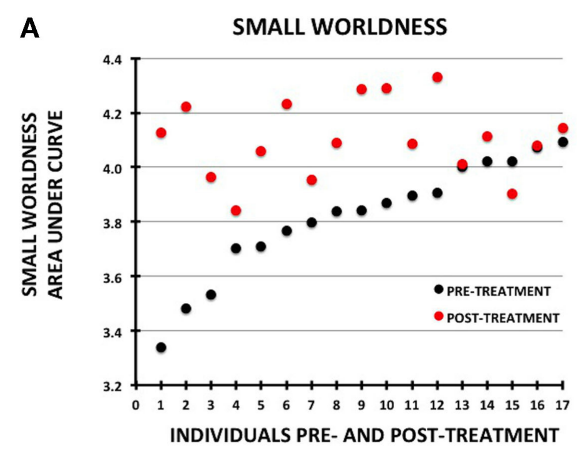

B

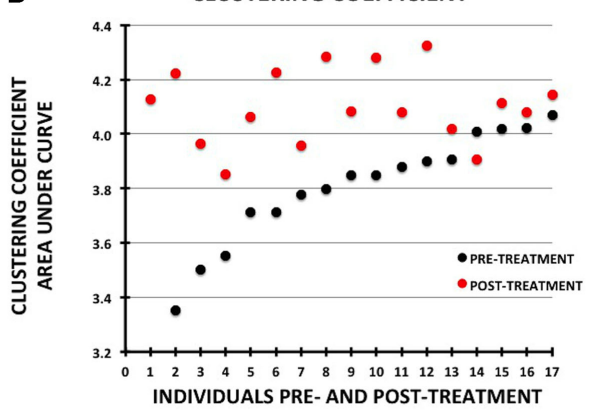

FIGURE 4 | Plots showing change in metrics for individual participants pre- vs. post-treatment. The data for each plot were sorted by the pre-treatment values. Pre-treatment values are shown with black circles and post-treatment as red circles: (A) small-worldness, (B) clustering coefficient. 


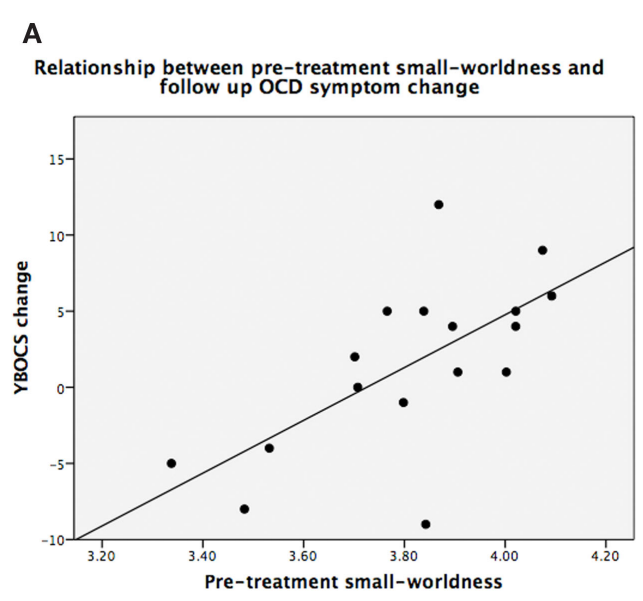

FIGURE 5 | (A) Scatterplot of small-worldness values vs. observed YBOCS changes in the post-treatment follow-up period; (B) linear regression results of predicted YBOCS changes vs. observed YBOCS changes. Note: (A) is
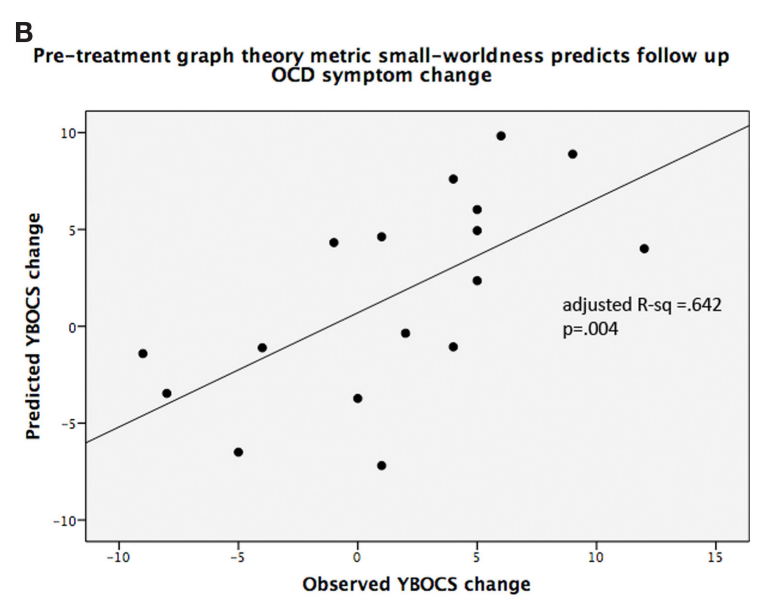

shown purely to display the relationship between the two variables, while (B) depicts the linear regression results that control for the covariates of non-interest. in effecting improvements in network efficiency could be independent from direct effects on obsessive thoughts and compulsive behaviors.

One possible mechanism could relate to general effects that CBT may have in helping patients organize their thoughts, feelings, and behaviors, and improve their ability to be a "rational observer" of these phenomena in their mind. This may relate to improvements through extended psychoeducation and practice in monitoring their experience, which can deepen their understanding of the mental processes inherent to OCD. Improvement in these areas, which provides the foundation and enhances insight to further address obsessions and compulsions with the behavioral (exposure and response prevention) stage of treatment, might confer increases in brain network efficiency. This aspect of CBT might allow for disparate, functionally segregated processing of emotions, thought patterns, sensory-motor experiences, and planned/executed behaviors to become more integrated into conscious awareness, which subsequently may be reflected in changes in small-world properties. Those with already high network efficiency when treatment begins may represent a more refractory group in the long-term, as they already may be employing these techniques but have not improved or remained well; this is the group that is at most risk for relapse. This conjectural interpretation, however, needs to be further explored and confirmed in future studies that could, for example, test effects of these components of psychoeducation and early stages of CBT, in isolation from its behavioral components.

Decreases in modularity with treatment were correlated with improvements in OCD symptoms. Optimal modularity implies an ideal number of communities in the network (58). Low modularity suggests relative isolation of certain nodes. Thus, CBT-associated decrease in modularity may reflect a declining influence of certain "pernicious" nodes (e.g., symptom-driving hyperactive frontostriatal loops) on network dynamics, which are associated with improvements in OCD symptoms. Alternatively, the observed reduction in modularity in response to $\mathrm{CBT}$ associated with reduction in OCD symptoms may result from remodeling of brain network modular structure (i.e., shifting membership of nodes in the various modules). Another possible explanation is that reductions in modularity values reflect the development of cognitive compensatory mechanisms allowing for greater control over obsessions and compulsions and hence improved symptoms; indirect evidence for this comes from a study that found that, within individuals, reductions in functional modularity were associated with enhanced working memory (59).

\section{Clinical Implications}

In our sample, small-world network efficiency was a biomarker for longitudinal outcome in OCD subsequent to CBT. The effectiveness of CBT is well documented and beyond doubt, as exemplified in the current study that produced a high proportion of responders and remitters. Hence, a more useful clinical question for the application of biomarkers is not who will respond to treatment, but rather who will remain well and/or continue to improve after treatment. rsfMRI is a relatively easy to perform procedure that could be administered and replicated across sites, lending this method both to larger trials to confirm its utility in providing network connectivity measures to assist with clinical decisionmaking, and, if confirmed, ultimately in actual use in clinical settings. Contrary to this, predictors that involve neuropsychological factors may be less reliable than other, objective measurements such as resting-state brain connectivity due to the fact that they are highly effort-dependent and in some cases evaluator-dependent. These findings may also set the stage for testing different treatment durations to reduce risk of relapse. Adequate studies have not been conducted to determine the duration of treatment optimal for preventing relapse (60).

\section{Limitations}

One limitation of this study is the small sample size, which may have constrained our ability to detect smaller changes in network measures and/or relationships with follow-up clinical status. This 
may have also accounted for the lack of significant node-level differences in graph-theory metrics pre- to post-treatment, for which we likely had insufficient power given the need to correct for multiple comparisons across 160 nodes. Another limitation, inherent to studies of a specific treatment modality that may restrict generalizability to the OCD population on the whole; it is possible that the OCD participants in this study may have "self-selected" for willingness to engage in difficult and intense exposure and response, which in turn likely results in significant reduction in symptoms.

\section{Conclusion}

Brain organization characterized by less dense local functional connections (lower clustering coefficient and therefore lower small-worldness) prior to CBT confers greater maintenance of treatment gains. CBT increases network efficiency as it alleviates

\section{References}

1. American Psychiatric Association. Diagnostic and Statistical Manual of Mental Disorders: DSM-5. Washington, DC: American Psychiatric Association (2013).

2. Markarian Y, Larson MJ, Aldea MA, Baldwin SA, Good D, Berkeljon A, et al. Multiple pathways to functional impairment in obsessive-compulsive disorder. Clin Psychol Rev (2010) 30:78-88. doi:10.1016/j.cpr.2009.09.005

3. Eddy KT, Dutra L, Bradley R, Westen D. A multidimensional meta-analysis of psychotherapy and pharmacotherapy for obsessive-compulsive disorder. Clin Psychol Rev (2004) 24:1011-30. doi:10.1016/j.cpr.2004.08.004

4. Calvocoressi L, McDougle CI, Wasylink S, Goodman WK, Trufan SJ, Price LH. Inpatient treatment of patients with severe obsessive-compulsive disorder. Hosp Community Psychiatry (1993) 44:1150-4.

5. Bystritsky A, Munford PR, Rosen RM, Martin KM, Vapnik T, Gorbis EE, et al. A preliminary study of partial hospital management of severe obsessivecompulsive disorder. Psychiatr Serv (1996) 47:170-4. doi:10.1176/ps.47.2.170

6. Meyer V. Modification of expectations in cases with obsessional rituals. Behav Res Ther (1966) 4:273-80. doi:10.1016/0005-7967(66)90023-4

7. Steketee G, Foa EB, Grayson JB. Recent advances in the behavioral treatment of obsessive-compulsives. Arch Gen Psychiatry (1982) 39:1365-71. doi:10.1001/ archpsyc.1982.04290120001001

8. Kirk JW. Behavioural treatment of obsessional-compulsive patients in routine clinical practice. Behav Res Ther (1983) 21:57-62. doi:10.1016/0005-7967(83) 90126-2

9. O’Sullivan G, Noshirvani H, Marks I, Monteiro W, Lelliott P. Six-year follow-up after exposure and clomipramine therapy for obsessive compulsive disorder. $J$ Clin Psychiatry (1991) 52:150-5.

10. Riggs D, Foa EB. Obsessive compulsive disorder. 2nd ed. In: Barlow DH, editor. Clinical Handbook of Psychological Disorders: A Step-by-Step Treatment Manual. New York, NY: Guilford Press (1993). p. 189-239.

11. Hiss H, Foa EB, Kozak MJ. Relapse prevention program for treatment of obsessive-compulsive disorder. J Consult Clin Psychol (1994) 62:801-8. doi:10. 1037/0022-006X.62.4.801

12. Neziroglu F, Henricksen J, Yaryura-Tobias JA. Psychotherapy of obsessivecompulsive disorder and spectrum: established facts and advances, 1995-2005. Psychiatr Clin North Am (2006) 29:585-604. doi:10.1016/j.psc.2006.02.004

13. Foa EB, Kozak MJ. Psychological treatment for obsessive - compulsive disorder. In: Mavissakalian M, Prien R, editors. Long-Term Treatments of Anxiety Disorders. Washington, DC: American Psychiatric Press, Inc. (1996). p. 285-309.

14. Simpson HB, Franklin ME, Cheng J, Foa EB, Liebowitz MR. Standard criteria for relapse are needed in obsessive-compulsive disorder. Depress Anxiety (2005) 21:1-8. doi:10.1002/da.20052

15. Ravizza L, Barzega G, Bellino S, Bogetto F, Maina G. Drug treatment of obsessive-compulsive disorder (OCD): long-term trial with clomipramine and symptoms in most patients, but patients entering CBT with already high-network efficiency are at greater risk of relapse. Results show functional network efficiency as a biomarker of CBT response and relapse in $\mathrm{OCD}$, which has potential implications for clinical decision-making and treatment selection. These results warrant further investigation and replication in a larger dataset.

\section{Acknowledgments}

The research was funded by NIMH grant R01MH085900 (JO and JF).

\section{Supplementary Material}

The Supplementary Material for this article can be found online at http://journal.frontiersin.org/article/10.3389/fpsyt.2015.00074/ abstract

selective serotonin reuptake inhibitors (SSRIs). Psychopharmacol Bull (1996) 32:167-73

16. Eisen JL, Goodman WK, Keller MB, Warshaw MG, Demarco LM, Luce DD, et al. Patterns of remission and relapse in obsessive-compulsive disorder: a 2year prospective study. J Clin Psychiatry (1999) 60:346-51. doi:10.4088/JCP. v60n0514

17. Maina G, Albert U, Bogetto F. Relapses after discontinuation of drug associated with increased resistance to treatment in obsessive-compulsive disorder. Int Clin Psychopharmacol (2001) 16:33-8. doi:10.1097/00004850-200101000-00004

18. Romano S, Goodman W, Tamura R, Gonzales J. Long-term treatment of obsessive-compulsive disorder after an acute response: a comparison of fluoxetine versus placebo. J Clin Psychopharmacol (2001) 21:46-52. doi:10.1097/ 00004714-200102000-00009

19. Simpson HB, Liebowitz MR, Foa EB, Kozak MJ, Schmidt AB, Rowan V, et al. Post-treatment effects of exposure therapy and clomipramine in obsessivecompulsive disorder. Depress Anxiety (2004) 19:225-33. doi:10.1002/da.20003

20. Keeley ML, Storch EA, Merlo LJ, Geffken GR. Clinical predictors of response to cognitive-behavioral therapy for obsessive-compulsive disorder. Clin Psychol $\operatorname{Rev}(2008)$ 28:118-30. doi:10.1016/j.cpr.2007.04.003

21. Raffin AAL, Ferrão YA, Souza FPD, Cordioli AV. Fatores preditores de resultados no tratamento do transtorno obsessivo-compulsivo com as terapias comportamental e cognitivo-comportamental: uma revisão sistemática. Rev Psiquiatr Rio Grande Sul (2008) 30. doi:10.1590/S0101-81082008000200006

22. Knopp J, Knowles S, Bee P, Lovell K, Bower P. A systematic review of predictors and moderators of response to psychological therapies in OCD: do we have enough empirical evidence to target treatment? Clin Psychol Rev (2013) 33:1067-81. doi:10.1016/j.cpr.2013.08.008

23. Eisen JL, Pinto A, Mancebo MC, Dyck IR, Orlando ME, Rasmussen SA. A 2 year prospective follow-up study of the course of obsessive-compulsive disorder. J Clin Psychiatry (2010) 71:1033-9. doi:10.4088/JCP.08m04806blu

24. Lewin AB, Wu MS, McGuire JF, Storch EA. Cognitive behavior therapy for obsessive-compulsive and related disorders. Psychiatr Clin North Am (2014) 37:415-45. doi:10.1016/j.psc.2014.05.002

25. Jakubovski E, Diniz JB, Valerio C, Fossaluza V, Belotto-Silva C, Gorenstein $\mathrm{C}$, et al. Clinical predictors of long-term outcome in obsessive-compulsive disorder. Depress Anxiety (2013) 30:763-72. doi:10.1002/da.22013

26. Braga DT, Manfro GG, Niederauer K, Cordioli AV. Full remission and relapse of obsessive-compulsive symptoms after cognitive-behavioral group therapy: a two-year follow-up. Rev Bras Psiquiatr (2010) 32:164-8. doi:10.1590/ s1516-44462010000200012

27. Stewart SE, Geller DA, Jenike M, Pauls D, Shaw D, Mullin B, et al. Longterm outcome of pediatric obsessive-compulsive disorder: a meta-analysis and qualitative review of the literature. Acta Psychiatr Scand (2004) 110:4-13. doi:10. 1111/j.1600-0447.2004.00302.x

28. Rufer M, Hand I, Alsleben H, Braatz A, Ortmann J, Katenkamp B, et al. Long-term course and outcome of obsessive-compulsive patients after 
cognitive-behavioral therapy in combination with either fluvoxamine or placebo: a 7-year follow-up of a randomized double-blind trial. Eur Arch Psychiatry Clin Neurosci (2005) 255:121-8. doi:10.1007/s00406-004-0544-8

29. Maia TV, Cooney RE, Peterson BS. The neural bases of obsessive-compulsive disorder in children and adults. Dev Psychopathol (2008) 20:1251-83. doi:10. 1017/S0954579408000606

30. Menzies L, Chamberlain SR, Laird AR, Thelen SM, Sahakian BJ, Bullmore ET. Integrating evidence from neuroimaging and neuropsychological studies of obsessive-compulsive disorder: the orbitofronto-striatal model revisited. Neurosci Biobehav Rev (2008) 32:525-49. doi:10.1016/j.neubiorev.2007.09.005

31. van den Heuvel OA, Veltman DJ, Groenewegen HJ, Cath DC, Van Balkom AJ, Van Hartskamp J, et al. Frontal-striatal dysfunction during planning in obsessive-compulsive disorder. Arch Gen Psychiatry (2005) 62:301-9. doi:10. 1001/archpsyc.62.3.301

32. Stern ER, Fitzgerald KD, Welsh RC, Abelson JL, Taylor SF. Resting-state functional connectivity between fronto-parietal and default mode networks in obsessive-compulsive disorder. PLoS One (2012) 7:e36356. doi:10.1371/journal. pone. 0036356

33. Bullmore E, Sporns O. Complex brain networks: graph theoretical analysis of structural and functional systems. Nat Rev Neurosci (2009) 10:186-98. doi:10. $1038 / \mathrm{nrn} 2575$

34. Zhang T, Wang J, Yang Y, Wu Q, Li B, Chen L, et al. Abnormal small-world architecture of top-down control networks in obsessive-compulsive disorder. $J$ Psychiatry Neurosci (2011) 36:23-31. doi:10.1503/jpn.100006

35. Shin DJ, Jung WH, He Y, Wang J, Shim G, Byun MS, et al. The effects of pharmacological treatment on functional brain connectome in obsessive-compulsive disorder. Biol Psychiatry (2014) 75:606-14. doi:10.1016/j.biopsych.2013.09.002

36. American Psychiatric Association. Diagnostic and Statistical Manual of Mental Disorders: DSM-IV-TR. Washington, DC: American Psychiatric Association (2000).

37. DiNardo PA, Brown TA, Barlow DH. Anxiety Disorders Interview Schedule for DSM-IV: Lifetime Version. Albany, NY: Graywind (1994).

38. Goodman WK, Price LH, Rasmussen SA, Mazure C, Fleischmann RL, Hill CL, et al. The Yale-Brown Obsessive Compulsive Scale. 1. Development, use, and reliability. Arch Gen Psychiatry (1989) 46:1006-11. doi:10.1001/archpsyc.1989. 01810110054008

39. Wechsler D. Wechsler Abbreviated Scale of Intelligence (WASI). San Antonio, TX: Psychological Corporation (1999).

40. Farris SG, McLean CP, Van Meter PE, Simpson HB, Foa EB. Treatment response, symptom remission, and wellness in obsessive-compulsive disorder. J Clin Psychiatry (2013) 74:685-90. doi:10.4088/JCP.12m07789

41. Hamilton M. The assessment of anxiety states by rating. Br J Med Psychol (1959) 32:50-5. doi:10.1111/j.2044-8341.1959.tb00467.x

42. Montgomery SA, Asberg M. A new depression scale designed to be sensitive to change. Br J Psychiatry (1979) 134:382-9. doi:10.1192/bjp.134.4.382

43. Endicott J, Spitzer RL, Fleiss JL, Cohen J. The global assessment scale. A procedure for measuring overall severity of psychiatric disturbance. Arch Gen Psychiatry (1976) 33:766-71. doi:10.1001/archpsyc.1976.01770060086012

44. Golden JC. Stroop Color and Word Test: A Manual for Clinical and Experimental Uses. Chicago, IL: Stoelting Company (1978).

45. Sheehan D. The Anxiety Disease. New York, NY: Scribner (1983).

46. Endicott J, Nee J, Harrison W, Blumenthal R. Quality of life enjoyment and satisfaction questionnaire: a new measure. Psychopharmacol Bull (1993) 29: $321-6$.
47. Power JD, Barnes KA, Snyder AZ, Schlaggar BL, Petersen SE. Spurious but systematic correlations in functional connectivity MRI networks arise from subject motion. Neuroimage (2012) 59:2142-54. doi:10.1016/j.neuroimage. 2011.10.018

48. Dosenbach NU, Nardos B, Cohen AL, Fair DA, Power JD, Church JA, et al. Prediction of individual brain maturity using fMRI. Science (2010) 329:1358-61. doi:10.1126/science.1194144

49. Yan CG, Craddock RC, He Y, Milham MP. Addressing head motion dependencies for small-world topologies in functional connectomics. Front Hum Neurosci (2013) 7:910. doi:10.3389/fnhum.2013.00910

50. Bullmore E, Sporns O. The economy of brain network organization. Nat Rev Neurosci (2012) 13(5):336-49. doi:10.1038/nrn3214

51. Sporns O. Networks of the Brain. Cambridge, MA: MIT Press (2010).

52. Das TK, Abeyasinghe PM, Crone JS, Sosnowski A, Laureys S, Owen AM, et al. Highlighting the structure-function relationship of the brain with the Ising model and graph theory. Biomed Res Int (2014) 2014:237898. doi:10.1155/2014/ 237898

53. Hollander E, Stein DJ, Fineberg NA, Marteau F, Legault M. Quality of life outcomes in patients with obsessive-compulsive disorder: relationship to treatment response and symptom relapse. JClin Psychiatry (2010) 71:784-92. doi:10.4088/ JCP.09m05911blu

54. Watts DJ, Strogatz SH. Collective dynamics of 'small-world' networks. Nature (1998) 393:440-2. doi:10.1038/30918

55. Latora V, Marchiori M. Efficient behavior of small-world networks. Phys Rev Lett (2001) 87:198701. doi:10.1103/PhysRevLett.87.198701

56. Stam CJ, Reijneveld JC. Graph theoretical analysis of complex networks in the brain. Nonlinear Biomed Phys (2007) 1:3. doi:10.1186/1753-4631-1-3

57. Rubinov M, Sporns O. Complex network measures of brain connectivity: uses and interpretations. Neuroimage (2010) 52:1059-69. doi:10.1016/j.neuroimage. 2009.10.003

58. Telesford QK, Simpson SL, Burdette JH, Hayasaka S, Laurienti PJ. The brain as a complex system: using network science as a tool for understanding the brain. Brain Connect (2011) 1:295-308. doi:10.1089/brain.2011.0055

59. Stanley ML, Dagenbach D, Lyday RG, Burdette JH, Laurienti PJ. Changes in global and regional modularity associated with increasing working memory load. Front Hum Neurosci (2014) 8:954. doi:10.3389/fnhum. 2014.00954

60. Pallanti S, Hollander E, Bienstock C, Koran L, Leckman J, Marazziti D, et al. Treatment non-response in OCD: methodological issues and operational definitions. Int J Neuropsychopharmacol (2002) 5:181-91. doi:10.1017/ S1461145702002900

Conflict of Interest Statement: The authors declare that the research was conducted in the absence of any commercial or financial relationships that could be construed as a potential conflict of interest.

Copyright (C) 2015 Feusner, Moody, Lai, Sheen, Khalsa, Brown, Levitt, Alger and O'Neill. This is an open-access article distributed under the terms of the Creative Commons Attribution License (CC BY). The use, distribution or reproduction in other forums is permitted, provided the original author(s) or licensor are credited and that the original publication in this journal is cited, in accordance with accepted academic practice. No use, distribution or reproduction is permitted which does not comply with these terms. 